\title{
Simple and Efficient Preconditioner for Surface Integral Solution of Scattering from Multilayer Dielectric Bodies
}

\author{
B. B. Kong and X. Q. Sheng \\ Center for Electromagnetic Simulation, School of Information and Electronics, Beijing Institute of Technology, Beijing 100081, China \\ Correspondence should be addressed to X. Q. Sheng; xsheng@bit.edu.cn
}

Received 20 April 2016; Revised 18 July 2016; Accepted 26 July 2016

Academic Editor: Lorenzo Crocco

Copyright ( 2016 B. B. Kong and X. Q. Sheng. This is an open access article distributed under the Creative Commons Attribution License, which permits unrestricted use, distribution, and reproduction in any medium, provided the original work is properly cited.

The computation of scattering from multilayer dielectric bodies is studied by using the combined tangential formulation (CTF) of surface integral solution. A simple and efficient preconditioner is designed for the surface integral solution of multilayer dielectric bodies and validated by numerical experiments. Compared with the traditional near field preconditioner, the proposed preconditioner significantly reduce CPU time and memory requirement. Furthermore, the multilevel fast multipole algorithm (MLFMA) is employed to improve the capability of the solutions. The trick of efficiently implementing MLFMA is presented for multilayer dielectric bodies. Numerical examples are presented to verify the accuracy and efficiency of the approach for computing scattering from multilayer dielectric problems.

\section{Introduction}

Electromagnetic scattering from multilayer dielectric bodies is an important problem in the computational electromagnetics (CEM) since it is widely used in real-life applications such as Luneburg lens, radomes, where each dielectric is fully included in the outer one. The method of moment (MoM) based on surface integral equations (SIE) has shown to be an efficient solution of this problem, since the volume discretization is avoided in this solution. The formulations of SIE have been well studied for homogeneous bodies in the literatures [1-5]. However, the numerical performance of surface integral solutions is not well investigated for multilayer dielectric bodies.

It is known that the preconditioners are usually required for obtaining an efficient surface integral solution of homogeneous bodies [6-8]. There are many preconditioners. A conventional preconditioner is constructed by directly employing the inverse of near field matrix, which is exactly compatible with the multilevel fast multipole algorithm (MLFMA). But it is very time-consuming and large memory requirement. Another general preconditioner is the block-diagonal preconditioner [9], which is constructed by choosing the block-diagonal part of the near-interaction.
However, it does not work well for CTF solution as verified in literature [5]. A more efficient preconditioner is the Schur complement preconditioner [7]. Since the discretized matrix of multilayer dielectric problems contains both the interactions of equivalent electric and magnetic currents on the same layer and those on different layers, it is hard to efficiently extend the Schur complement preconditioner from homogeneous bodies to multilayer bodies. In this letter, the combined tangential formulation (CTF) is employed to formulate scattering from multilayer dielectric bodies due to its high accuracy and efficient memory. Furthermore, CTF is free of internal resonances [4]. A simple preconditioner is designed for the efficient CTF solution of multilayer bodies. Compared with the existing preconditioners [6-8], it is easier to be implemented to the multilayer dielectric bodies. MLFMA $[10,11]$ is efficiently incorporated into the solution to improve its efficiency. Numerical experiments are presented to investigate the performance of the proposed approach.

\section{Theory and Formulation}

2.1. Surface Integral-Equation Formulations. Consider electromagnetic scattering from a multilayer dielectric object placed in free space and illuminated by an incident plane 


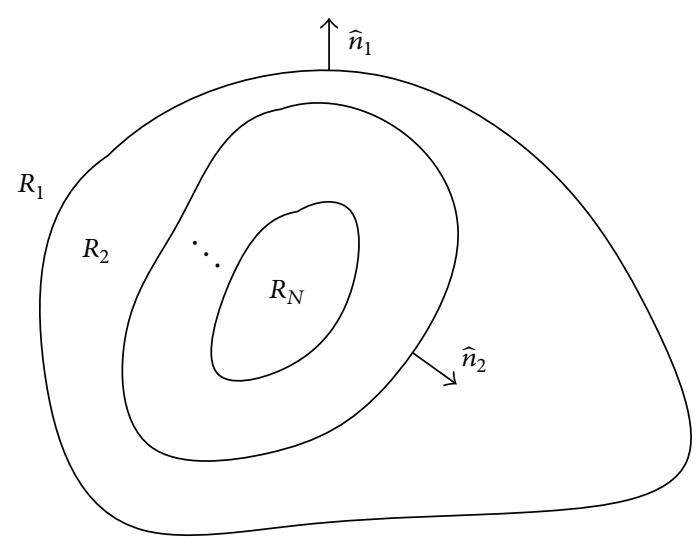

FIGURE 1: A multilayer dielectric scatterer with $N-1$ layer medium.

wave $\left(\mathbf{E}^{i}, \mathbf{H}^{i}\right)$. The exterior free space region is denoted as $R_{1}$ and the interior regions are denoted as $R_{i}$ with $i=2,3, \ldots, N$ for $N-1$ dielectric layers from outside to inside, with each dielectric being fully included in the outer one, as illustrated in Figure 1. The permittivity and permeability of region $R_{i}$ are $\varepsilon_{i}$ and $\mu_{i}$, respectively. The interface between two regions $R_{i}$ and $R_{i+1}$ is denoted by $S_{i}$ and $\widehat{n}_{i}$ denotes the unit normal of $S_{i}$ pointing toward the interior of $R_{i}$. The equivalent electric and magnetic currents on $S_{i}$ are denoted as $\mathbf{J}_{i}$ and $\mathbf{M}_{i}$, respectively.

The electric field integral equation in the region $R_{i}(i=$ $1,2,3, \ldots, N)$ can be formulated as [12]

$$
\begin{aligned}
\mathbf{E}_{i}= & \left(1-\delta_{1 i}\right) \\
& \cdot\left[Z_{i} \mathbf{L}_{i}\left(-\mathbf{J}_{i-1}\right)-\mathbf{K}_{i}\left(-\mathbf{M}_{i-1}\right)+\frac{\Omega}{4 \pi} \widehat{n}_{i-1} \times \mathbf{M}_{i-1}\right] \\
& +\left(1-\delta_{N i}\right)\left[Z_{i} \mathbf{L}_{i}\left(\mathbf{J}_{i}\right)-\mathbf{K}_{i}\left(\mathbf{M}_{i}\right)+\frac{\Omega}{4 \pi} \widehat{n}_{i} \times \mathbf{M}_{i}\right] \\
& +\delta_{1 i} \mathbf{E}^{i} .
\end{aligned}
$$

The magnetic field integral equation in the region $R_{i}$ can be formulated as

$$
\begin{aligned}
\mathbf{H}_{i}= & \left(1-\delta_{1 i}\right) \\
& \cdot\left[\frac{1}{Z_{i}} \mathbf{L}_{i}\left(-\mathbf{M}_{i-1}\right)+\mathbf{K}_{i}\left(-\mathbf{J}_{i-1}\right)-\frac{\Omega}{4 \pi} \widehat{n}_{i-1} \times \mathbf{J}_{i-1}\right] \\
& +\left(1-\delta_{N i}\right)\left[\frac{1}{Z_{i}} \mathbf{L}_{i}\left(\mathbf{M}_{i}\right)+\mathbf{K}_{i}\left(\mathbf{J}_{i}\right)-\frac{\Omega}{4 \pi} \widehat{n}_{i} \times \mathbf{J}_{i}\right] \\
& +\delta_{1 i} \mathbf{H}^{i},
\end{aligned}
$$

where $Z_{i}=\sqrt{\mu_{i} / \varepsilon_{i}},\left(\mathbf{E}_{i}, \mathbf{H}_{i}\right)$ denote the total field in $R_{i}$ and $\Omega$ is the solid angle which equals $2 \pi$ for common smooth surface. $\delta_{j i}=1$ for $i=j$ and $\delta_{j i}=0$ for other cases. The operators $\mathbf{L}$ and $\mathbf{K}$ are defined as

$$
\begin{aligned}
& \mathbf{L}_{i}(\mathbf{X})=-j k_{i} \int\left[\mathbf{X}+\frac{1}{k_{i}^{2}} \nabla\left(\nabla^{\prime} \cdot \mathbf{X}\right)\right] G_{i} d r^{\prime}, \\
& \mathbf{K}_{i}(\mathbf{X})=\int_{\mathrm{PV}} \mathbf{X} \times \nabla^{\prime} G_{i} d r^{\prime} .
\end{aligned}
$$

Here PV indicates the principal value of the integral, $G_{i}$ is Green's function in region $R_{i}$, and $k_{i}=\omega \sqrt{\varepsilon_{i} \mu_{i}}$.

By combining the equations in region $R_{i}$ with those in region $R_{i+1}$, we obtain the following CTF formulation [5]:

$$
\begin{aligned}
\widehat{t} \cdot & {\left[\mathbf{L}_{i}+\mathbf{L}_{i+1}\right]\left(\mathbf{J}_{i}\right)-\hat{t} } \\
\cdot & {\left[\frac{1}{Z_{i}}\left(\mathbf{K}_{i}+\frac{1}{2} \mathbf{I}_{n}\right)+\frac{1}{Z_{i+1}}\left(\mathbf{K}_{i+1}-\frac{1}{2} \mathbf{I}_{n}\right)\right]\left(\mathbf{M}_{i}\right) } \\
& -\left(1-\delta_{N i}\right)\left[\widehat{t} \cdot \mathbf{L}_{i+1}\left(\mathbf{J}_{i+1}\right)-\widehat{t} \cdot \frac{1}{Z_{i+1}} \mathbf{K}_{i+1}\left(\mathbf{M}_{i+1}\right)\right] \\
& -\left(1-\delta_{1 i}\right)\left[\widehat{t} \cdot \mathbf{L}_{i}\left(\mathbf{J}_{i-1}\right)-\widehat{t} \cdot \frac{1}{Z_{i}} \mathbf{K}_{i}\left(\mathbf{M}_{i-1}\right)\right]=\delta_{1 i} e_{i}, \\
\widehat{t} \cdot & {\left[Z_{i}\left(\mathbf{K}_{i}+\frac{1}{2} \mathbf{I}_{n}\right)+Z_{i+1}\left(\mathbf{K}_{i+1}-\frac{1}{2} \mathbf{I}_{n}\right)\right]\left(\mathbf{J}_{i}\right)+\widehat{t} } \\
& \cdot\left[\mathbf{L}_{i}+\mathbf{L}_{i+1}\right]\left(\mathbf{M}_{i}\right) \\
& -\left(1-\delta_{N i}\right)\left[\widehat{t} \cdot Z_{i+1} \mathbf{K}_{i+1}\left(\mathbf{J}_{i+1}\right)+\widehat{t} \cdot \mathbf{L}_{i+1}\left(\mathbf{M}_{i+1}\right)\right] \\
& -\left(1-\delta_{1 i}\right)\left[\hat{t} \cdot Z_{i} \mathbf{K}_{i}\left(\mathbf{J}_{i-1}\right)+\widehat{t} \cdot \mathbf{L}_{i}\left(\mathbf{M}_{i-1}\right)\right]=\delta_{1 i} h_{i},
\end{aligned}
$$

where $i=1,2,3, \ldots, N-1$, the operator $\mathbf{I}_{n}\left(\mathbf{X}_{i}\right)=\widehat{n}_{i} \times \mathbf{X}_{i}$, and $e_{1}=-\left(1 / Z_{1}\right) \hat{t} \cdot \mathbf{E}^{i}, h_{1}=-Z_{1} \hat{t} \cdot \mathbf{H}^{i}$.

\subsection{Discretization of Surface Integral-Equation Formulations.} To discretize (4) and (5), each dielectric surface/interface $S_{i}$ is meshed by planner triangular patches. The surface currents are expanded with Rao-Wilton-Glisson (RWG) basis functions [13]. Applying Galerkin's testing method, we obtain the following discretized matrix equation:

$$
\begin{aligned}
{\left[\begin{array}{cccccccc}
\mathbf{A}_{1} & \mathbf{U}_{1} & 0 & 0 & \cdots & 0 & 0 & 0 \\
\mathbf{Q}_{2} & \mathbf{A}_{2} & \mathbf{U}_{2} & 0 & \cdots & 0 & 0 & 0 \\
0 & \mathbf{Q}_{3} & \mathbf{A}_{3} & \mathbf{U}_{3} & \cdots & 0 & 0 & 0 \\
\vdots & \ddots & \ddots & \ddots & \ddots & \ddots & \ddots & \vdots \\
0 & 0 & 0 & 0 & \cdots & \mathbf{Q}_{N-2} & \mathbf{A}_{N-2} & \mathbf{U}_{N-2} \\
0 & 0 & 0 & 0 & \cdots & 0 & \mathbf{Q}_{N-1} & \mathbf{A}_{N-1}
\end{array}\right]\left(\begin{array}{c}
x_{1} \\
x_{2} \\
x_{3} \\
\vdots \\
x_{N-2} \\
x_{N-1}
\end{array}\right) } \\
=\left(\begin{array}{c}
b_{1} \\
0 \\
0 \\
\vdots \\
0 \\
0
\end{array}\right),
\end{aligned}
$$

where $x_{i}=\left(J_{i}, M_{i}\right)^{T}$ and $J_{i}$ and $M_{i}$ are the unknowns of electric and magnetic currents, respectively. To be more 
specific, the submatrices of $\mathbf{A}_{i}, \mathbf{U}_{i}, \mathbf{Q}_{i}, b_{1}$ have the following structures:

$$
\begin{aligned}
\mathbf{A}_{i} & =\left[\begin{array}{ll}
\mathbf{A}_{i}^{1}+\mathbf{A}_{i}^{2} & \mathbf{A}_{i}^{3}+\mathbf{A}_{i}^{4} \\
\mathbf{A}_{i}^{5}+\mathbf{A}_{i}^{6} & \mathbf{A}_{i}^{7}+\mathbf{A}_{i}^{8}
\end{array}\right], \\
\mathbf{U}_{i} & =\left[\begin{array}{ll}
\mathbf{U}_{i}^{1} & \mathbf{U}_{i}^{2} \\
\mathbf{U}_{i}^{3} & \mathbf{U}_{i}^{4}
\end{array}\right], \\
\mathbf{Q}_{i} & =\left[\begin{array}{ll}
\mathbf{Q}_{i}^{1} & \mathbf{Q}_{i}^{2} \\
\mathbf{Q}_{i}^{3} & \mathbf{Q}_{i}^{4}
\end{array}\right], \\
b_{1} & =\left[\begin{array}{c}
b_{1}^{e} \\
b_{1}^{m}
\end{array}\right],
\end{aligned}
$$

where

$$
\begin{aligned}
& \mathbf{A}_{i}^{1}=\left\langle\mathbf{g}, \mathbf{L}_{i}(\mathbf{g})\right\rangle, \\
& \mathbf{A}_{i}^{2}=\left\langle\mathbf{g}, \mathbf{L}_{i+1}(\mathbf{g})\right\rangle, \\
& \mathbf{A}_{i}^{3}=\left\langle\mathbf{g},-\frac{1}{Z_{i}}\left(\mathbf{K}_{i}+\frac{1}{2} \mathbf{I}_{n}\right)(\mathbf{g})\right\rangle, \\
& \mathbf{A}_{i}^{4}=\left\langle\mathbf{g},-\frac{1}{Z_{i+1}}\left(\mathbf{K}_{i+1}-\frac{1}{2} \mathbf{I}_{n}\right)(\mathbf{g})\right\rangle, \\
& \mathbf{A}_{i}^{5}=\left\langle\mathbf{g}, Z_{i}\left(\mathbf{K}_{i}+\frac{1}{2} \mathbf{I}_{n}\right)(\mathbf{g})\right\rangle, \\
& \mathbf{A}_{i}^{6}=\left\langle\mathbf{g}, Z_{i+1}\left(\mathbf{K}_{i+1}-\frac{1}{2} \mathbf{I}_{n}\right)(\mathbf{g})\right\rangle, \\
& \mathbf{A}_{i}^{7}=\left\langle\mathbf{g}, \mathbf{L}_{i}(\mathbf{g})\right\rangle, \\
& \mathbf{A}_{i}^{8}=\left\langle\mathbf{g}, \mathbf{L}_{i+1}(\mathbf{g})\right\rangle, \\
& \mathbf{U}_{i}^{1}=\left\langle\mathbf{g},-\mathbf{L}_{i+1}(\mathbf{g})\right\rangle \text {, } \\
& \mathbf{U}_{i}^{2}=\left\langle\mathbf{g}, \frac{1}{Z_{i+1}} \mathbf{K}_{i+1}(\mathbf{g})\right\rangle, \\
& \mathbf{U}_{i}^{3}=\left\langle\mathbf{g},-Z_{i+1} \mathbf{K}_{i+1}(\mathbf{g})\right\rangle, \\
& \mathbf{U}_{i}^{4}=\left\langle\mathbf{g},-\mathbf{L}_{i+1}(\mathbf{g})\right\rangle, \\
& \mathbf{Q}_{i}^{1}=\left\langle\mathbf{g},-\mathbf{L}_{i}(\mathbf{g})\right\rangle, \\
& \mathbf{Q}_{i}^{2}=\left\langle\mathbf{g}, \frac{1}{Z_{i}} \mathbf{K}_{i}(\mathbf{g})\right\rangle, \\
& \mathbf{Q}_{i}^{3}=\left\langle\mathbf{g},-Z_{i} \mathbf{K}_{i}(\mathbf{g})\right\rangle, \\
& \mathbf{Q}_{i}^{4}=\left\langle\mathbf{g},-\mathbf{L}_{i}(\mathbf{g})\right\rangle, \\
& b_{1}^{e}=\left\langle\mathbf{g},-\frac{1}{Z_{1}} \mathbf{E}^{i}\right\rangle, \\
& b_{1}^{m}=\left\langle\mathbf{g},-Z_{1} \mathbf{H}^{i}\right\rangle,
\end{aligned}
$$

where $\mathbf{g}$ denotes the RWG basis and testing functions on $S_{i}$ and $\langle\cdot, \cdot\rangle$ stands for the inner product.

\section{Implementation of MLFMA for Multilayer Bodies}

To efficiently solve (7), MLFMA is employed to speed up the matrix-vector multiplication in iterative solutions. Usually, each sub-matrix-vector multiplication of (7) is independently speeded up by using MLFMA. Each dielectric layer shares the same cubic clusters, but different truncation numbers in MLFMA are employed. However, if the special structure of (9) is employed, a more efficient MLFMA implementation can be achieved. For example, since the far-field interactions of $\mathbf{A}_{i}^{1}$ and $\mathbf{A}_{i}^{5}$ in (7) act on the same equivalent electric currents and also Green's function for $\mathbf{A}_{i}^{1}$ and $\mathbf{A}_{i}^{5}$ is the same, the aggregation and translation components for their far-field interactions are the same; only the receiving components are different. Thus, the calculation of the far-field interactions of $\mathbf{A}_{i}^{1}$ and $\mathbf{A}_{i}^{5}$ in each matrix-vector multiple of the iteration can be finished with only once computation of the aggregation and translation and twice disaggregations. The same trick can be applied to $\mathbf{A}_{i}^{2}$ and $\mathbf{A}_{i}^{6}, \mathbf{A}_{i}^{3}$ and $\mathbf{A}_{i}^{7}, \mathbf{A}_{i}^{4}$ and $\mathbf{A}_{i}^{8}$, $\mathbf{U}_{i}^{1}$ and $\mathbf{U}_{i}^{3}$, $\mathbf{U}_{i}^{2}$ and $\mathbf{U}_{i}^{4}, \mathbf{Q}_{i}^{1}$ and $\mathbf{Q}_{i}^{3}$, and $\mathbf{Q}_{i}^{2}$ and $\mathbf{Q}_{i}^{4}$.

\section{Construction of Preconditioner}

Preconditioners are usually required for iterative solutions of (7) since the increase of the number of dielectric layers leads to the rapid growth of the condition number of impedance matrix. Employing a preconditioner on the matrix equation of (7) yields

$$
\mathbf{P}^{-1} \mathbf{M} x=\mathbf{P}^{-1} b .
$$

A conventional preconditioner is constructed by employing the inverse of the near field matrix, which is called near field preconditioner (NFP). NFP is exactly compatible with MLFMA. However, the construction of NFP is timeconsuming and large memory is required. More efficient preconditioners are proposed by making use of the approximate Schur complement [7]. Since (7) is not a $2 \times 2$ partitioned system $[14,15]$, these preconditioners are difficult to be extended from homogeneous bodies to multilayer dielectric object.

In this letter, a simple preconditioner is designed for the iterative solution of (7). In the construction of NFP, the near field matrix is obtained by maintaining the entries of selfinteraction in one box and near-interaction with the neighbor boxes and omitting other entries of the original matrix. The boxes are ones in the lowest level of MLFMA. Since the number of the entries of near-interaction is quite large, NFP is resource-consuming, especially for multilayer dielectric targets since multilayer dielectric bodies have interactions between equivalent currents on different interfaces. To overcome such drawbacks, we employ the interaction distances $L$ of entries between source and field as criterion to determine whether the entries are retained or omitted, as shown in

$$
\mathbf{P}_{i j}= \begin{cases}\mathbf{M}_{i j} & \left|\mathbf{r}_{i}-\mathbf{r}_{j}\right| \leq L \\ 0 & \text { elsewhere }\end{cases}
$$




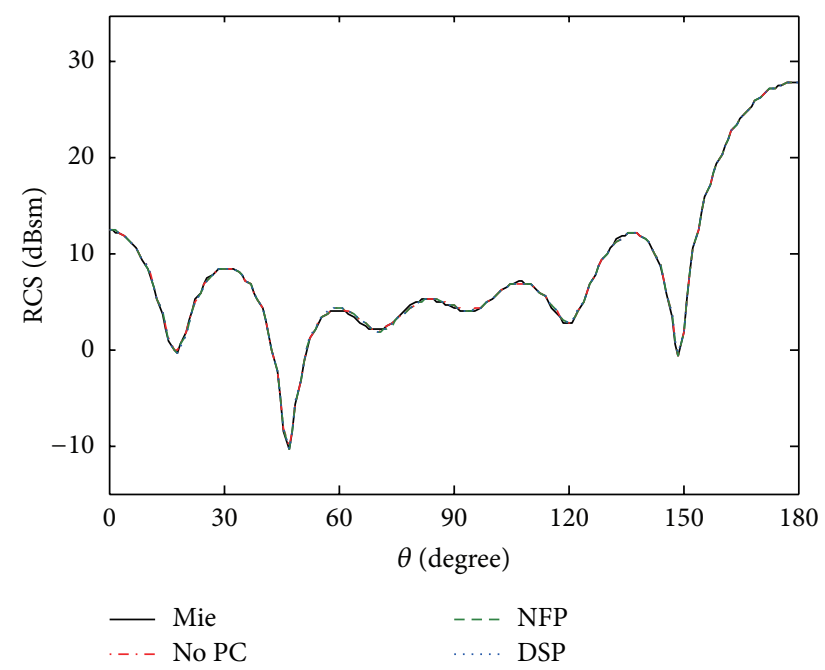

FIGURE 2: $\theta \theta$-polarized bistatic RCS of a multilayer sphere.

where $\mathbf{r}_{i}$ and $\mathbf{r}_{j}$ represent the location of the testing and bases elements. To be more specific, if the interaction distance between source and field is less than $0.15 \lambda_{0}$, the matrix entries are retained; otherwise the matrix entries are omitted. Here $\lambda_{0}$ is the free space wavelength. For multilayer bodies, the distance between source and basis functions is important because the interactions between different layers are stronger when the layers are more close to each other. The maintained entries include interactions between different layers if the distance between layers is small. Thus the sparsified scheme can retain the main interactions between source and basis functions. Since only those entries within self-elements and neighbor elements are maintained in this sparsified scheme, the number of the maintained entries is much smaller than that in NFP. We employ the inverse of this sparsified matrix as preconditioner and call it distance sparse preconditioner (DSP).

\section{Numerical Results}

In this section, we present several examples to demonstrate the accuracy and efficiency of the presented approach. All the simulations are at frequency of $300 \mathrm{MHz}$. The generalized minimum residual (GMRES) solver is used and a residual error of $10^{-3}$ is set to terminate iterations. MUMPS is used to calculate the inverse of the sparsified matrix in the preconditioner construction. MUMPS, short for "Multifrontal Massively Parallel Solver," is a direct sparse solver based on the parallel multifrontal method [16].

First, we consider a dielectric multilayer body consisting of 5 concentric spheres with radius of $0.6 \lambda_{0}, 0.7 \lambda_{0}, 0.8 \lambda_{0}$, $0.9 \lambda_{0}$, and $\lambda_{0}$ for each interface from inside to outside. The relative permittivity of regions $R_{2}, R_{3}, R_{4}, R_{5}$, and $R_{6}$ is 3,2, 3, 2 , and 1 , respectively. The body is illuminated by a plane wave propagating in $z$ direction with the electric field polarized in $x$ direction. The body is meshed with $\lambda_{0} / 10$ element size and has 16,128 unknowns. Figure 2 presents $\theta \theta$-polarized bistatic RCS in $x z$-plane for this multilayer sphere. It shows that

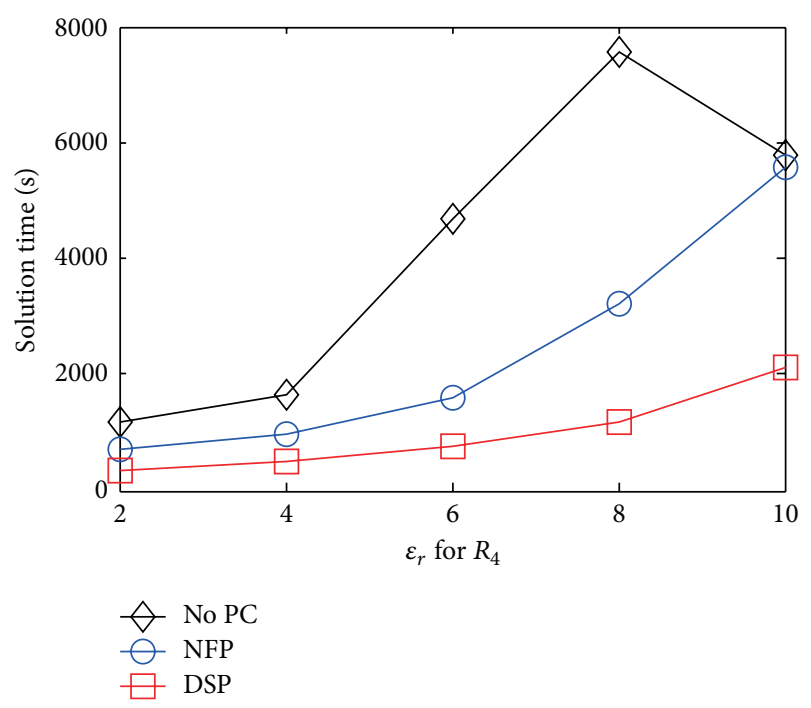

FIGURE 3: Total solution time as a function of $\varepsilon_{r}$ under different preconditioner.

the numerical results agree well with the analytical Mieseries solution. The numerical performance of MLFMA with NFP and DSP and no-preconditioner (No PC) is presented in Table 1. The CPU time and the memory requirement in Table 1 are those for the analysis and factorization step of MUMPS for constructing the inverse matrix of the preconditioners. It can be seen that iteration number is greatly reduced by using preconditioners. The total time required by DSP is only about $1 / 4$ of that required by NFP. Furthermore, the memory required by DSP is less than $1 / 5$ of that required by NFP. In addition, the efficient MLFMA implementation presented in this letter can reduce the iteration CPU time from 2,000 s required by the conventional MLFMA implementation to $1,060 \mathrm{~s}$.

To investigate the numerical performance of the solution for bodies with higher dielectric constant, the same multilayer sphere but with different permittivity $\varepsilon_{r}$ of the layer $R_{4}$ is calculated. The parameters of $\varepsilon_{r}$ are listed in Table 2. The element sizes used in the meshing $S_{3}$ and $S_{4}$ with different $\varepsilon_{r}$ are shown in Table 2. The resulted numbers of unknowns are also presented in Table 2. The total solution time as a function of $\varepsilon_{r}$ is plotted in Figure 3. It can be seen that DSP has the least total solution time for all dielectric constants. Furthermore, DSP has more advantage over NFP with higher dielectric constant.

The next three examples are computed to further demonstrate the capability of the presented approach for multilayer dielectric objects with different shapes, compared with the commercial software of FEKO. The first object is a cubic shell having three dielectric layers of $\varepsilon_{r}=2,3,4$ from inside to outside. Each of the layers has a thickness of $0.1 \lambda_{0}$ and the side length of the cubic shell is $4 \lambda_{0}$, as shown in Figure $4(\mathrm{a})$. The average mesh size is $0.1 \lambda_{0}$ and the number of unknowns is 122,544 . The bistatic RCS patterns for $\theta \theta$ - and $\varphi \varphi$-polarizations are computed. Figures 4(b) and 4(c) present the comparison of bistatic RCS obtained by DSP with the results from $\mathrm{FEKO}$, where the mesh size is also set at $0.1 \lambda_{0}$ and 


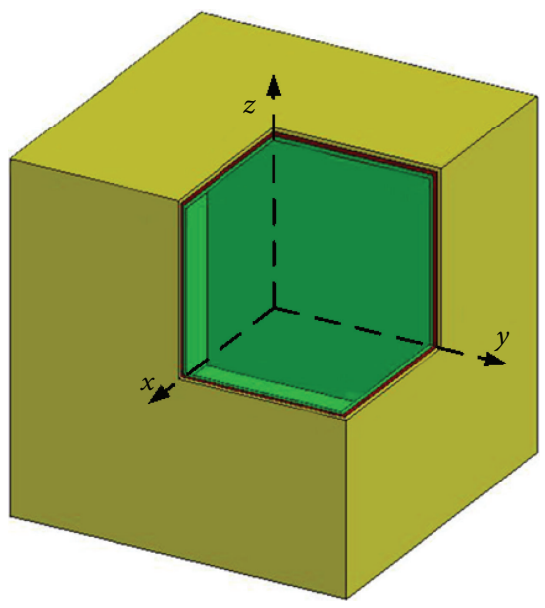

(a)

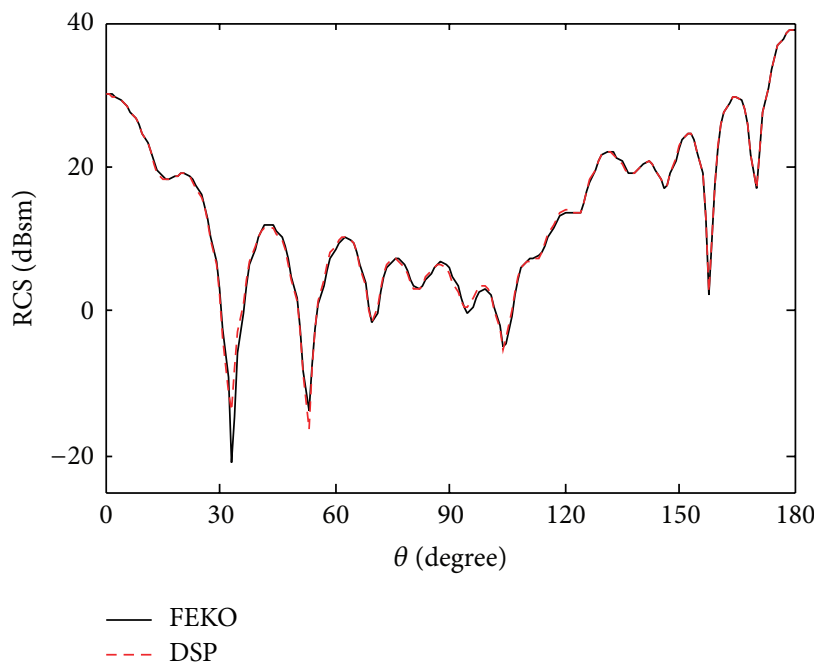

(b)

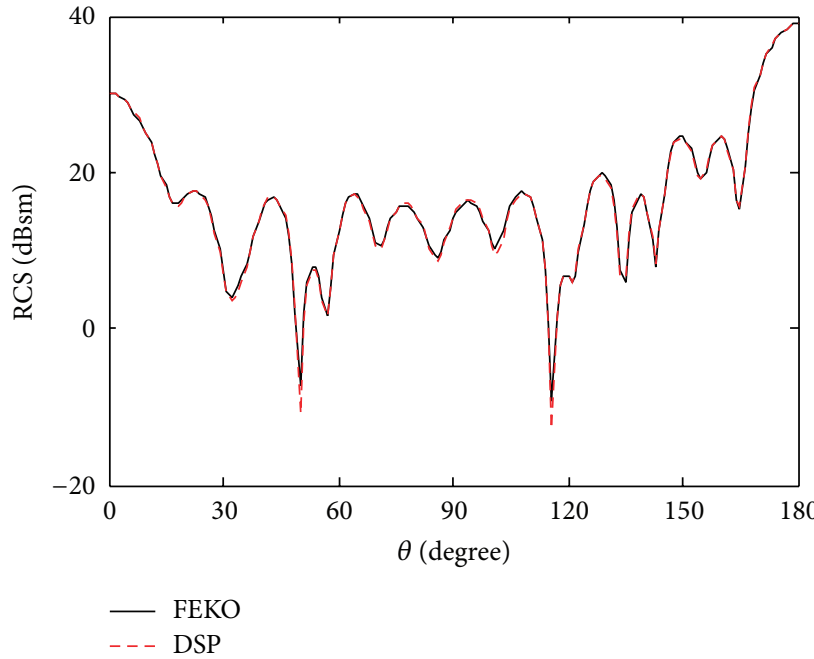

(c)

FIgURE 4: (a) Cubic shell consists of three dielectric layers. (b) $\theta \theta$-polarized bistatic RCS. (c) $\varphi \varphi$-polarized bistatic RCS.

TABLE 1: Comparison of numerical performances of NFP, DSP, and No PC for the multilayer sphere.

\begin{tabular}{lccccc}
\hline & CPU time for PC (s) & Memory for PC (MB) & Iteration number & Iteration time (s) & Total time (s) \\
\hline No PC & - & - & 513 & 1,060 & 1,060 \\
NFP & 445 & 3,693 & 15 & 47 & 492 \\
DSP & 34 & 673 & 45 & 105 & 139 \\
\hline
\end{tabular}

TABLE 2: Parameters of the multilayer spherical shell.

\begin{tabular}{lcc}
\hline$\varepsilon_{r}$ of $R_{4}$ & Element size in meshing $S_{3}, S_{4}$ & Number of unknowns \\
\hline 2 & $0.1 \lambda_{0}$ & 16,128 \\
4 & $0.08 \lambda_{0}$ & 19,299 \\
6 & $0.07 \lambda_{0}$ & 22,014 \\
8 & $0.06 \lambda_{0}$ & 26,163 \\
10 & $0.05 \lambda_{0}$ & 33,012 \\
\hline
\end{tabular}

the residual error is $10^{-3}$. It is shown that the computational values of DSP are in agreement with results from FEKO.
The second example is an ellipsoidal shell with radius along $x$-, $y$-, and $z$-axis being $2 \lambda_{0}, 2 \lambda_{0}$, and $\lambda_{0}$, respectively, as shown in Figure 5(a). It consists of three dielectric layers of $\varepsilon_{r}=2,3,4$ from inside to outside. The thickness of the innermost layer is $0.2 \lambda_{0}$ and the others are both $0.05 \lambda_{0}$. Bistatic RCS obtained using DSP with 45,558 unknowns is compared with the result from FEKO in Figures 5(b) and 5(c) and good agreement is observed.

The last example is a cylindrical cavity as shown in Figure 6(a), which is a concave object. The radius of the cylindrical cavity at the base is $2.0 \lambda_{0}$ and the height is $4.0 \lambda_{0}$. It consists of three dielectric layers; each has thickness of 


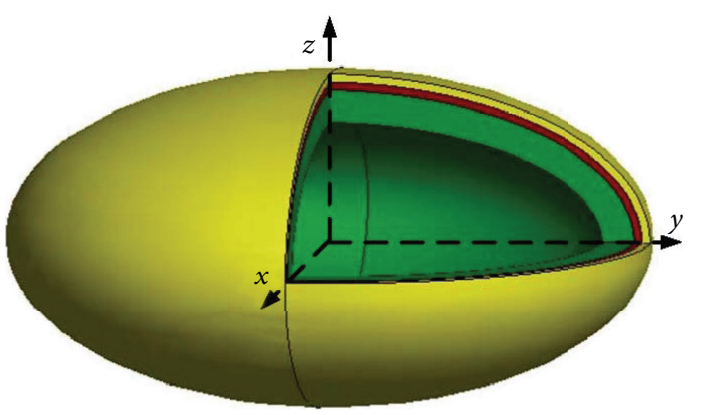

(a)

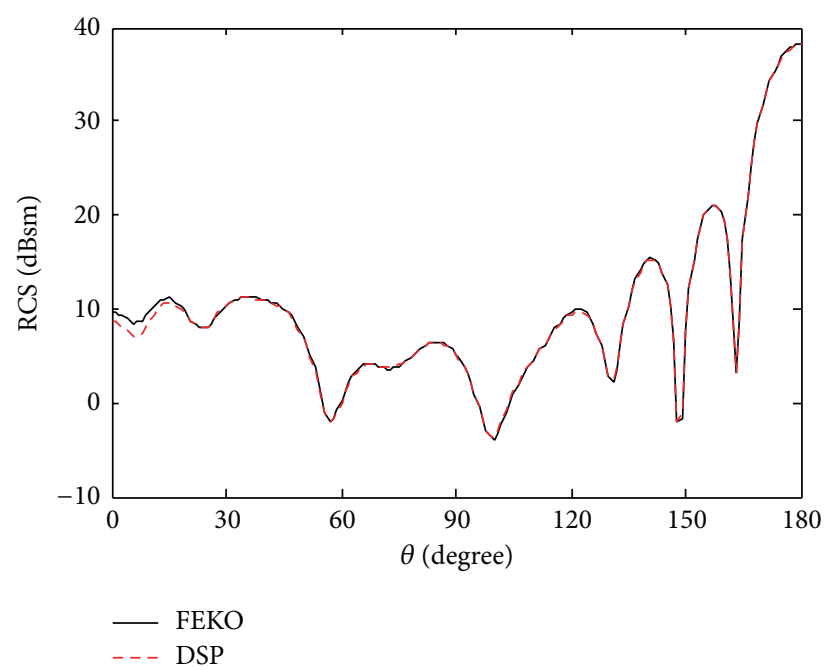

(b)

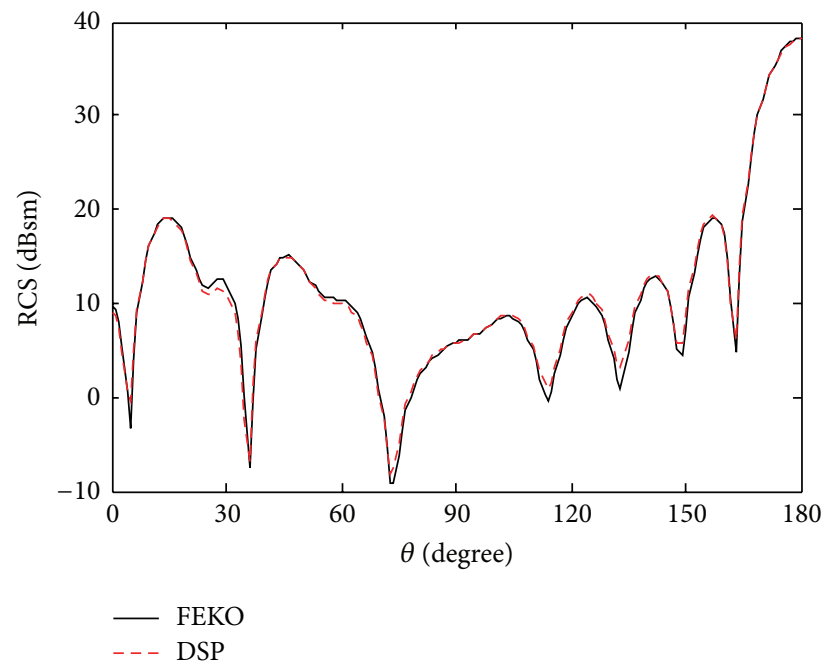

(c)

FIGURE 5: (a) Ellipsoidal shell with three dielectric layers. (b) $\theta \theta$-polarized bistatic RCS. (c) $\varphi \varphi$-polarized bistatic RCS.

$0.1 \lambda_{0}$. The middle layer has $\varepsilon_{r}=2$ and the other two layers have $\varepsilon_{r}=3$. Bistatic RCS obtained using DSP with 80,250 unknowns is compared with the result from FEKO in Figures 6(b) and 6(c) and good agreement is observed. The computation information of these three examples is listed in Table 3. The presented solution is more efficient than FEKO.

\section{Conclusions}

The surface integral solution of scattering from multilayer dielectric bodies is studied by using CTF. A simple preconditioner of DSP is presented, and its efficiency is validated by numerical experiments. Compared with the conventional near field preconditioner, the proposed preconditioner of DSP greatly reduce computation time and memory requirement. An efficient MLFMA implementation is given for multilayer dielectric bodies. Numerical results show that the
TABLE 3: Comparison of total solution time and peak memory of DSP and FEKO for the scattering problems.

\begin{tabular}{lccc}
\hline Problem & Method & Total solution time (s) & Peak memory (MB) \\
\hline \multirow{2}{*}{ Figure 4 } & FEKO & 9,582 & 42,812 \\
& DSP & 2,680 & 14,789 \\
\hline \multirow{2}{*}{ Figure 5 } & FEKO & 2,742 & 12,481 \\
& DSP & 1,283 & 4,543 \\
\hline \multirow{2}{*}{ Figure 6 } & FEKO & 24,448 & 24,090 \\
& DSP & 2,480 & 11,212 \\
\hline
\end{tabular}

presented MLFMA implementation can save half iteration time compared with the conventional MLFMA implementation. Numerical results show that the presented CTF solution is much more efficient than FEKO for multilayer dielectric bodies. 


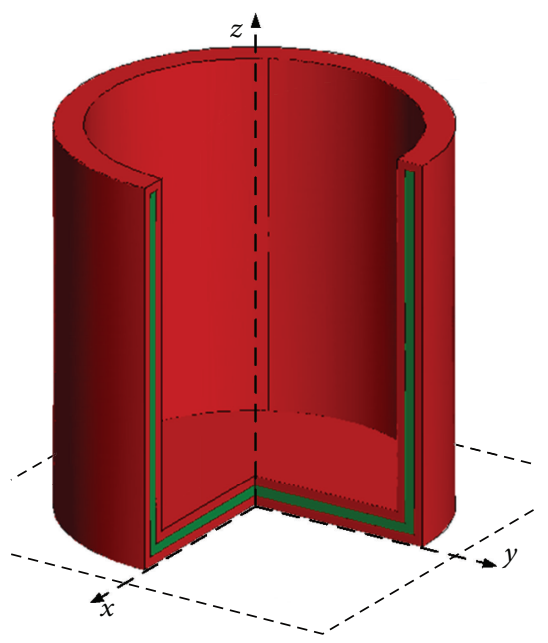

(a)

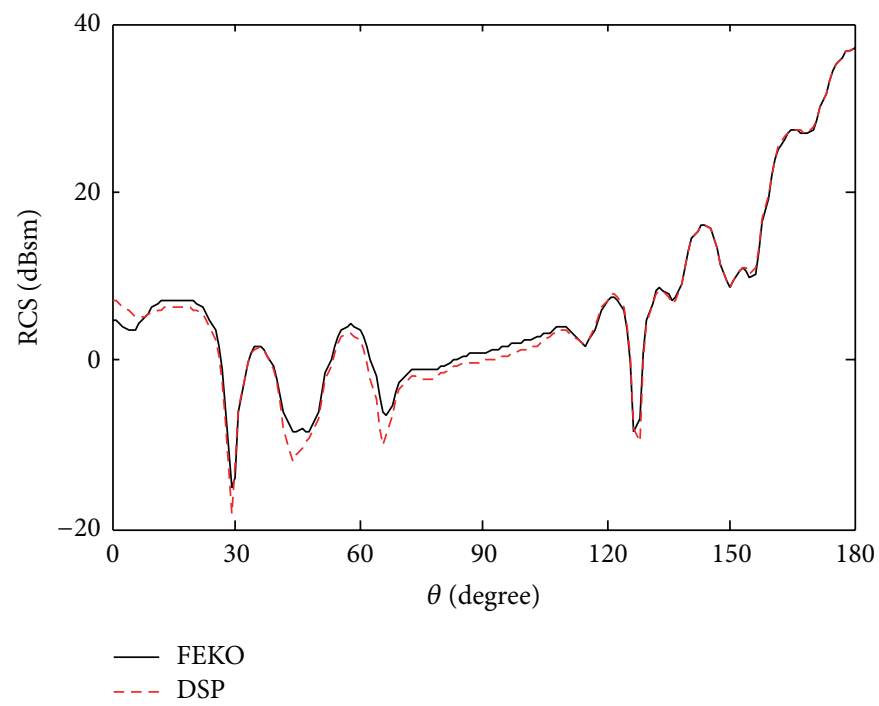

(b)

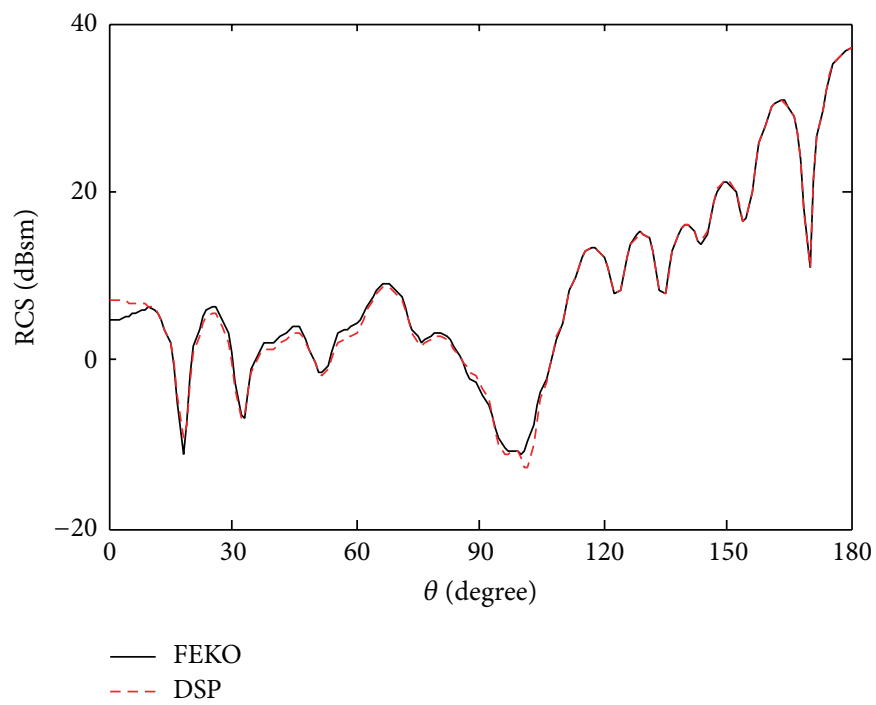

(c)

FIgURE 6: (a) Cylindrical cavity with three dielectric layers. (b) $\theta \theta$-polarized bistatic RCS. (c) $\varphi \varphi$-polarized bistatic RCS.

\section{Competing Interests}

The authors declare that there is no conflict of interests regarding the publication of this paper.

\section{Acknowledgments}

This work was supported by the National Basic Research Program (973) under Grants no. 61320602 and no. 61327301, the 111 Project of China under Grant B14010, and the NSFC under Grant no. 61421001.

\section{References}

[1] T.-K. Wu and L. L. Tsai, "Scattering from arbitrarily-shaped lossy dielectric bodies of revolution," Radio Science, vol. 12, no. 5, pp. 709-718, 1977.
[2] R. F. Harrington, "Boundary integral formulations for homogeneous material bodies," Journal of Electromagnetic Waves and Applications, vol. 3, no. 1, pp. 1-15, 1989.

[3] S. M. Rao and D. R. Wilton, "E-Field, H-Field, and combined field solution for arbitrarily shaped three-dimensional dielectric bodies," Electromagnetics, vol. 10, no. 4, pp. 407-421, 1990.

[4] P. Ylä-Oijala, M. Taskinen, and S. Järvenpää, "Surface integral equation formulations for solving electromagnetic scattering problems with iterative methods," Radio Science, vol. 40, no. 6 , p. S6002, 2005.

[5] Ö. Ergül and L. Gürel, "Comparison of integral-equation formulations for the fast and accurate solution of scattering problems involving dielectric objects with the multilevel fast multipole algorithm," IEEE Transactions on Antennas and Propagation, vol. 57, no. 1, pp. 176-187, 2009.

[6] S. Yan, J.-M. Jin, and Z. Nie, "A comparative study of Calderón preconditioners for PMCHWT equations," IEEE Transactions on Antennas and Propagation, vol. 58, no. 7, pp. 2375-2383, 2010. 
[7] T. Malas and L. Gürel, "Schur complement preconditioners for surface integral-equation formulations of dielectric problems solved with the multilevel fast multipole algorithm," SIAM Journal on Scientific Computing, vol. 33, no. 5, pp. 2440-2467, 2011.

[8] Ö. Ergül, T. Malas, and L. Gürel, "Analysis of dielectric photonic-crystal problems with MLFMA and Schurcomplement preconditioners," Journal of Lightwave Technology, vol. 29, no. 6, pp. 888-897, 2011.

[9] J. Song, C. C. Lu, and W. C. Chew, "Multilevel fast multipole algorithm for electromagnetic scattering by large complex objects," IEEE Transactions on Antennas and Propagation, vol. 45, no. 10, pp. 1488-1493, 1997.

[10] J. M. Song and W. C. Chew, "Multilevel fast-multipole algorithm for solving combined field integral equations of electromagnetic scattering," Microwave and Optical Technology Letters, vol. 10, no. 1, pp. 14-19, 1995.

[11] J.-S. Zhao and W. C. Chew, "Three-dimensional multilevel fast multipole algorithm from static to electrodynamic," Microwave and Optical Technology Letters, vol. 26, no. 1, pp. 43-48, 2000.

[12] K. C. Donepudi, J.-M. Jin, and W. C. Chew, "A higher order multilevel fast multipole algorithm for scattering from mixed conducting/dielectric bodies," IEEE Transactions on Antennas and Propagation, vol. 51, no. 10, pp. 2814-2821, 2003.

[13] S. M. Rao, D. R. Wilton, and A. W. Glisson, "Electromagnetic scattering by surfaces of arbitrary shape," IEEE Transactions on Antennas and Propagation, vol. 30, no. 3, pp. 409-418, 1982.

[14] J.-H. Yeom, H. Chin, H.-T. Kim, and K.-T. Kim, "Block matrix preconditioner method for the electric field integral equation (EFIE) formulation based on loop-star basis functions," Progress in Electromagnetics Research, vol. 134, pp. 543-558, 2013.

[15] Y.-Q. Wu, M.-J. Gou, and X.-Q. Sheng, "On the preconditioners of higher order method of moments for scattering by homogeneous dielectric objects," Journal of Electromagnetic Waves and Applications, vol. 26, no. 14-15, pp. 1809-1819, 2012.

[16] P. R. Amestoy, I. S. Duff, J.-Y. L'Excellent, and J. Koster, "A fully asynchronous multifrontal solver using distributed dynamic scheduling," SIAM Journal on Matrix Analysis and Applications, vol. 23, no. 1, pp. 15-41, 2001. 


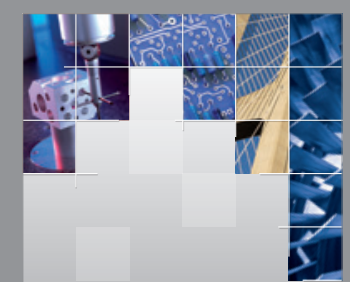

\section{Enfincering}
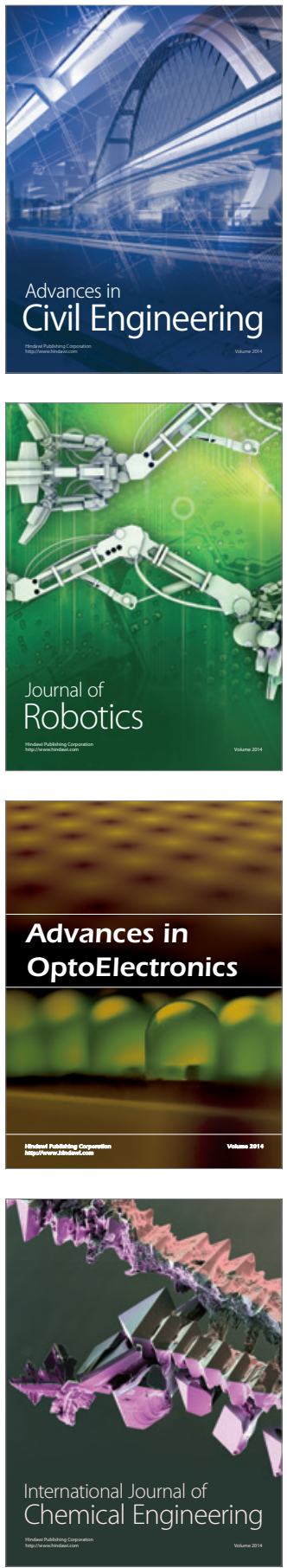

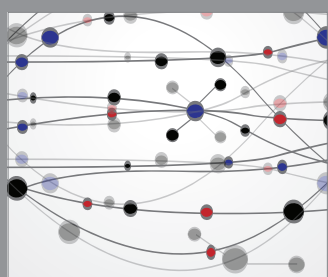

The Scientific World Journal

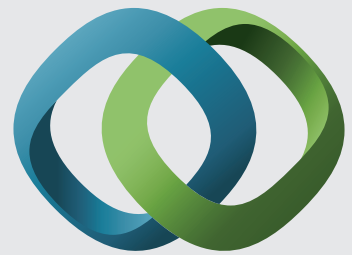

\section{Hindawi}

Submit your manuscripts at

http://www.hindawi.com
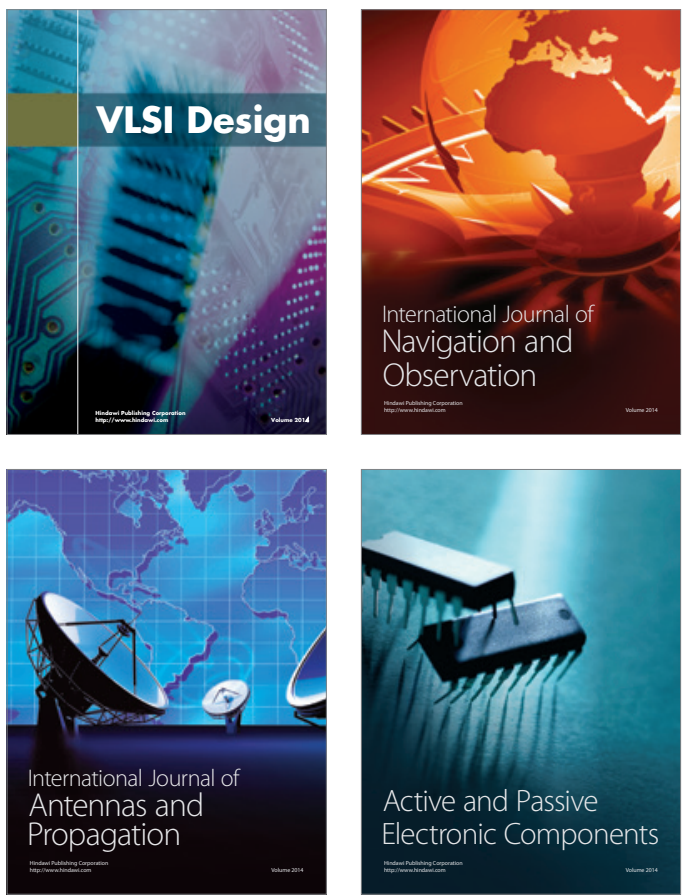
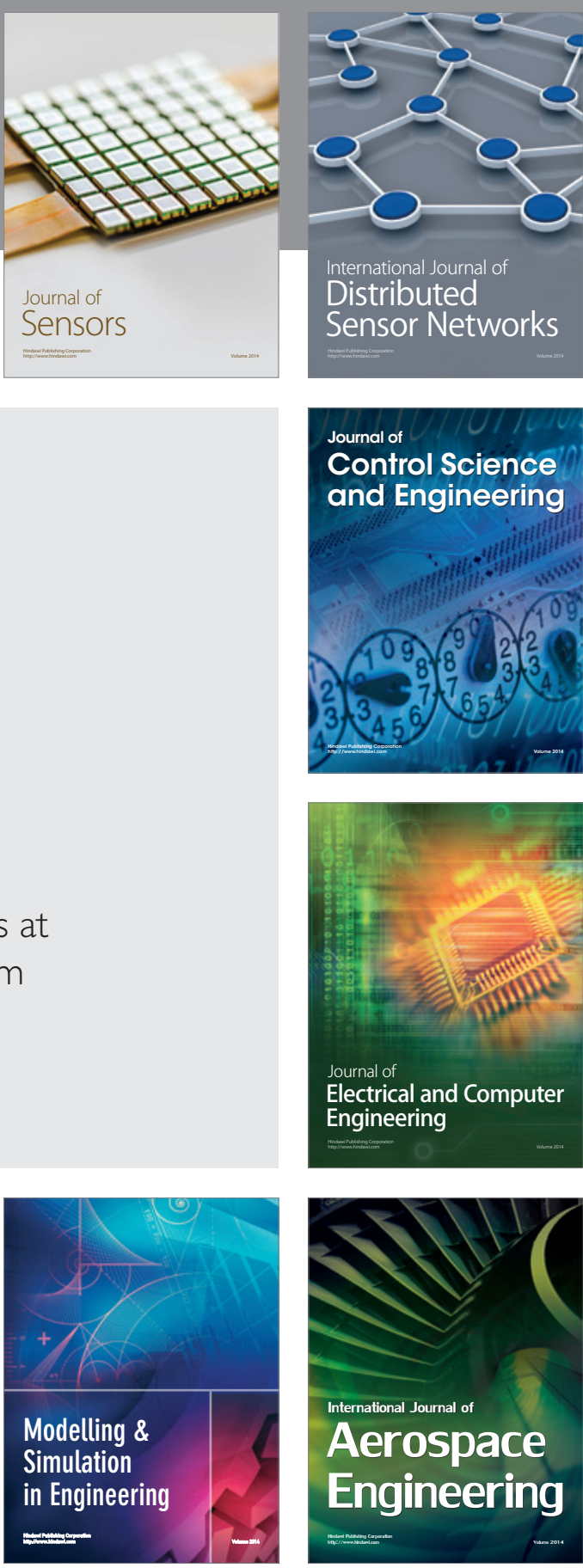

International Journal of

Distributed

Sensor Networks

Journal of

Control Science

and Engineering
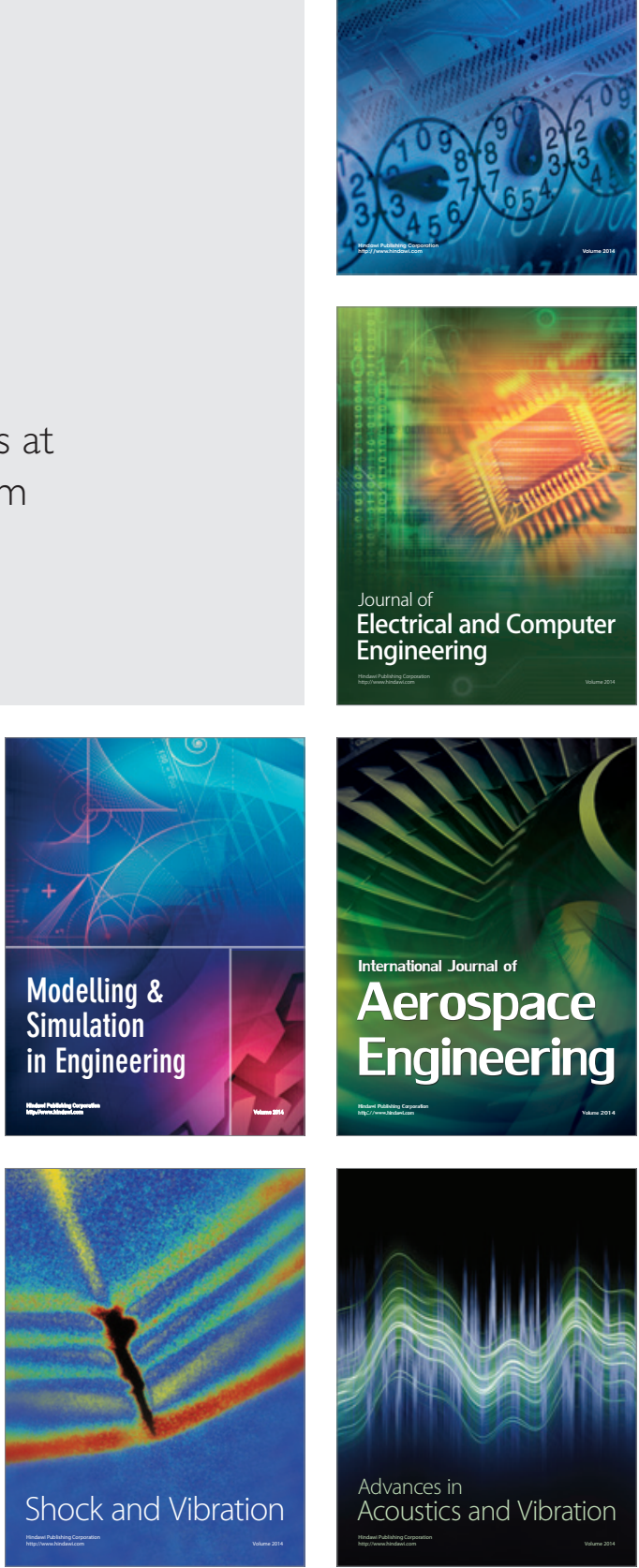